\title{
A Heuristic Algorithm for the Analysis of Fast Field Cycling (FFC) NMR Dispersion Curves
}

Paolo Lo Meo,* Samuele Terranova, Antonella Di Vincenzo, Delia Chillura Martino, Pellegrino Conte*

\author{
Supporting Information
}

1. Further details on the implementation of the heuristic algorithm

2. Experimental details

3. Table S1. Relaxometric data for the water-saturated commercial cellulose sample. 


\section{Further details on the implementation of the heuristic algorithm}

Relaxometric data processing was performed by setting up a "home-made" procedure, with the aid of the MS Excel software package. Basically, our mathematical problem relies in the resolution of equation 10, which can be performed by means of a numeric integration procedure. In other words, the expression:

$$
\int_{-\infty}^{\infty}\left[\left(\frac{10^{\lambda_{c}}}{1+\left(\omega \cdot 10^{\lambda_{c}}\right)^{2}}+\frac{4 \cdot 10^{\lambda_{c}}}{1+4\left(\omega \cdot 10^{\lambda_{c}}\right)^{2}}\right)\right]\left[l^{*}\left(\lambda_{c}\right)\right]^{2} d \lambda_{c}
$$

is discretely calculated as:

$$
\sum_{\lambda_{\min }}^{\lambda_{\max }}\left\{\left(\frac{10^{\lambda_{c}}}{1+\left(\omega \cdot 10^{\lambda_{c}}\right)^{2}}+\frac{4 \cdot 10^{\lambda_{c}}}{1+4\left(\omega \cdot 10^{\lambda_{c}}\right)^{2}}\right) \cdot\left[l^{*}\left(\lambda_{c}\right)\right]^{2} \cdot \delta \lambda_{c}\right\}
$$

In doing this, critical issues are the values for the limits $\lambda_{\max }$ and $\lambda_{\min }$ of the summation range and the summation step $\delta \lambda_{\mathrm{c}}$. The former ones are linked, in turn, to the range of $\omega$ values of the NMRD curve examined, i.e. 35 0.01 MHz. Such a range, in fact, is able to sample dynamic domains with $\tau_{\mathrm{c}}$ values ranging from 0.03 to $100 \mu \mathrm{s}$. Hence, as long as $\omega$ and $\tau_{\mathrm{c}}$ values are given in $\mathrm{MHz}$ and $\mu \mathrm{s}$ respectively, a reasonably large range for $\lambda_{c}$ values may be set between -3 and 3 , at a first instance (i.e. in the $\tau_{\mathrm{c}}$ range from 0.001 to $1000 \mu \mathrm{s}$ ). Regarding the step $\delta \lambda_{\mathrm{c}}$, its choice is trickier, because a compromise must be achieved between calculation accuracy and cpu time needed. After few trials, we found out that the best choice is constituted by a two-step procedure. First, $\delta \lambda_{c}$ value of 0.05 is used, integrating over the entire range chosen. In doing this, we verified that, after having completed the optimization procedure (i.e. after having minimized the parameter $S S Q^{*}$ parameter, according to the usual square residuals minimization criterion) the peaks featured in the obtained $\tau_{\mathrm{c}}$ spectrum are actually restricted in a significantly narrower $\lambda_{\mathrm{c}}$ range. Thus, we repeated the calculation/optimization procedure using a $\delta \lambda_{c}$ value of 0.02 and suitably restricting at the same time the $\lambda_{c}$ range. It is worth noting that the choice of $\delta \lambda_{c}=0.02 \log$ units corresponds to a variation in the corresponding $\tau_{\mathrm{c}}$ values just lower than the well accepted limit of 5\%. However, we verified 
that the number and the position of the peaks in the $\tau_{\mathrm{c}}$ spectra obtained are substantially independent of the step value used.

As we already mentioned, the entire regression analysis procedure was performed exploiting the MS Excel software package. In brief, after having defined the set of $\lambda_{c}$ values (depending on the chosen range and step) for the numerical integration of equation 10, we also defined a set of suitable starting values for the function $l^{*}\left(\lambda_{\mathrm{c}}\right)$, as well as suitable starting values for the offset $R_{0}$ and the parameters for the quadrupolar dips expression (if required). Therefore, we arranged a worksheet to calculate, for each $\omega_{\mathrm{L}}$ value of the experimental dataset, the relevant $R^{\mathrm{HH}}$ and $R^{\mathrm{NH}}$ contributions, then the value $R_{\text {cled }}\left(\omega_{\mathrm{L}}\right)=R_{0}+R^{\mathrm{HH}}+R^{\mathrm{NH}}$, and finally the $S S Q^{*}$ parameter defined by equation 11 (i.e. the canonical sum of square residuals). At this point we exploited the "Solver" module implemented in the Excel software package to simultaneously optimize the $l^{*}\left(\lambda_{\mathrm{c}}\right)$ function values, $R_{0}$ and the dips parameters. In doing this, a critical issue is again the choice of the initial $l^{*}\left(\lambda_{\mathrm{c}}\right)$ values. After few trials, we found out that a suitable solution is a Gaussian-like distribution function, i.e.:

$$
\operatorname{starting} l^{*}\left(\lambda_{c}\right)=C_{0} \cdot \exp \left[\left(\lambda_{0}-\lambda_{c}\right)^{2} / s\right]
$$

Here $\lambda_{0}$ is simply the center of the $\lambda_{\mathrm{c}}$ range, $C_{0}$ and $s$ are two constants, the value of which is arbitrarily fixed. We always verified that, whatever the initial set of fixed $l^{*}\left(\lambda_{c}\right)$ values is, the optimization procedure leads quite quickly to identify a restricted number of $\tau_{\mathrm{c}}$ domains, although actual convergence requires several thousands of steps.

In order to calculate the errors on the regression parameters, and in general to assess the possible presence of artifact peaks in the $\tau_{\mathrm{c}}$ spectrum, a Monte-Carlo procedure was implemented, according to the method proposed by Alper and Gelb for the analysis of thermodynamic data relevant to the study of the enthalpy-entropy compensation effect in supramolecular host-guest systems (J. Phys. Chem. 1990, 94, 4747-4751). In brief, for each sample virtual datasets are calculated starting from the experimental data, which are modified by applying them a Gaussianlike statistical error. In details, a set of $i$ casual numbers $\boldsymbol{n}_{\boldsymbol{i}}$ subjected to a Gaussian distribution is generated according to the formula:

$$
\boldsymbol{n}_{\boldsymbol{i}}=\left[-2 \cdot \ln \left(n_{1}\right)\right]^{1 / 2} \cdot \cos \left(2 \pi n_{2}\right)
$$


where $n_{1}$ and $n_{2}$ are two casual numbers between 0 and 1 . Then a virtual dataset is generated according to the equation:

$$
{ }^{\operatorname{virtual}} R_{\mathrm{i}}\left(\omega_{\mathrm{i}}\right)={ }^{\exp } R_{\mathrm{i}}\left(\omega_{\mathrm{i}}\right)+\boldsymbol{n}_{\boldsymbol{i}} \cdot \delta R_{\mathrm{i}}
$$

where $\exp _{\mathrm{i}}\left(\omega_{\mathrm{i}}\right)$ is a generic datum of the experimental dataset, and $\delta R_{\mathrm{i}}$ is the relevant experimental error (of course, different $\boldsymbol{n}_{\boldsymbol{i}}$ values are used for the different $R_{\mathrm{i}}\left(\omega_{\mathrm{i}}\right)$ values of the experimental dataset). The virtual dataset is then subjected to the heuristic analysis. For each sample a suitable ensemble of virtual dataset (up to 15) was generated. The relevant $\tau_{\mathrm{c}}$ spectra were superimposed, to identify clusters and possible artifacts. Then their sum was used as a cumulative probability function $\left[C P F\left(\tau_{\mathrm{c}}\right)\right]$, from which the standard deviation for each cluster was calculated as:

$$
\sigma\left(\tau_{c}\right)=\frac{\int_{a}^{b}\left(\tau_{c}-<\tau_{c}>\right)^{2}\left[C P F\left(\tau_{c}\right)\right] d \tau_{c}}{\int_{a}^{b}\left[C P F\left(\tau_{c}\right)\right] d \tau_{c}}
$$

Were $a$ and $b$ are suitable integration limits and $\left\langle\tau_{\mathrm{c}}\right\rangle$ is defined as:

$$
<\tau_{c}>=\frac{\int_{a}^{b} \tau_{c}\left[\operatorname{CPF}\left(\tau_{c}\right)\right] d \tau_{c}}{\int_{a}^{b}\left[C P F\left(\tau_{c}\right)\right] d \tau_{c}}
$$




\section{Experimental details.}

The synthesis and characterization of the cyclodextrin nanosponge has been described in details elsewhere (see J. Phys. Chem. B. 2020, 124 (9), 1847-1857, and references therein). In brief, the proper amounts of dry $\beta$-cyclodextrin and hexamethylene-bis-isocyanate (1:4 mole/mole) were mechanically mixed in the presence of the minimum amount of dry DMSO, and the mixture was kept overnight at $60{ }^{\circ} \mathrm{C}$. The product is repeatedly washed to eliminate the DMSO, dried and crunched in a ball mill. Details on the Parmigiano Reggiano cheese sample can be found in Food Res. Int. 2020, 10984. Cristalline commercial cellulose (Sigma) was mixed with milliQ grade water $(1: 1 \mathrm{w} / \mathrm{w})$, and kept overnight before performing FFC-NMR analysis.

FFC NMR experiments were performed on a Stelar Spinmaster FFC 2000 relaxometer (Stelar s.r.l., Mede, PV- Italy) at the constant temperature of $25^{\circ} \mathrm{C}$. When pre-polarization was needed, the proton spins were polarized at a polarization field $\left(\mathrm{B}_{\mathrm{POL}}\right)$ corresponding to a proton Larmor frequency $\left(v_{\mathrm{L}}\right)$ of $10 \mathrm{MHz}$ for a period of polarization corresponding to ca. four times the $T_{1}$ estimated at this frequency. After each $\mathrm{B}_{\mathrm{POL}}$ application, the magnetic field intensity (indicated as $B_{\mathrm{RLX}}$ ) was systematically changed through the proton Larmor frequency range 9-0.01 MHz. When the pre-polarization was not needed, $B_{P O L}$ was null, while $B_{R L X}$ was varied in the $v_{L}$ range 35-9 MHz. The period $\tau$, during which $B_{R L X}$ was applied, was varied on 32 logarithmic spaced time sets, each of them adjusted at every relaxation field in order to optimize the sampling of the decay/recovery curves. FIDs were recorded following a single ${ }^{1} \mathrm{H} 90^{\circ}$ pulse of $5.5 \mu$ s applied at an acquisition field corresponding to the proton Larmor frequency of 7.20 MHz. A time domain of $100 \mu$ s sampled with 1000 points was applied. Field-switching time was 3 ms, while spectrometer dead time was $15 \mu \mathrm{s}$. For all the experiments, a recycle delay of $1 \mathrm{~s}$ was used. 
3. Table S1. Relaxometric data for the water-saturated commercial cellulose sample.

\begin{tabular}{ll}
\hline$\omega_{\mathrm{L}}(\mathrm{MHz})$ & $R\left(\mathrm{~s}^{-1}\right)$ \\
\hline 10.000 & $1.29 \pm 0.04$ \\
8.000 & $1.41 \pm 0.04$ \\
6.000 & $1.63 \pm 0.05$ \\
5.000 & $1.79 \pm 0.06$ \\
4.000 & $1.96 \pm 0.06$ \\
3.600 & $2.09 \pm 0.08$ \\
3.000 & $2.19 \pm 0.07$ \\
2.000 & $2.62 \pm 0.09$ \\
1.000 & $3.58 \pm 0.09$ \\
0.800 & $3.92 \pm 0.11$ \\
0.600 & $4.20 \pm 0.10$ \\
0.300 & $5.32 \pm 0.13$ \\
0.090 & $7.58 \pm 0.15$ \\
0.050 & $9.26 \pm 0.18$ \\
0.010 & $16.03 \pm 0.17$ \\
\hline
\end{tabular}

